\title{
B2-EIRENE modelling of He compression and enrichment
}

\author{
Coster, D.P., Bosch, H.-S., Ullrich, W. and the ASDEX Upgrade team ${ }^{\mathrm{a}}$ \\ a Max-Planck-Institut für Plasmaphysik, EURATOM Association, D-85748 Garching, Germany
}

\begin{abstract}
Helium compression and helium enrichment are important for most future fusion reactors since the He ash produced by the nuclear reactions needs to be pumped out to prevent its accumulation in the core of the plasma. (Helium compression is the ratio of the helium density in the pumping plenum to the helium density in the plasma; helium enrichment is the ratio of the helium compression to the hydrogen or deuterium compression.) In this work the B2-Eirene code is used to model helium compression and enrichment on ASDEX Upgrade with divertor II under a variety of plasma densities, input powers, transport coefficients and divertor geometries so as to determine the range of acceptable operating conditions for effective He removal. A broad range of acceptable plasma conditions is found, though a lower limit of the upstream separatrix density is noted.
\end{abstract}

\section{Introduction}

Helium compression (the ratio of the density of helium in the exhaust stream to the helium density in edge plasma) is important for future reactor machines (such as ITER) because the helium ash has to be removed at a sufficient rate so that the thermonuclear reaction can continue without being quenched by the accumulation of helium. Helium enrichment (the ratio of helium compression to the compression of the working gas) is also important for the design of the tritium handling plant and the breeding blanket since the helium is exhausted with a $\mathrm{D} / \mathrm{T}$ mixture and this exhaust stream has to be separated out into its components[1].

In this work the B2-Eirene code package[2,3] has been used to simulate helium transport in the edge plasma of ASDEX Upgrade. B2-Eirene is the combination of a multi-fluid plasma code (B2)[4,5] (following all the charged states of hydrogen, carbon and helium) with a Monte-Carlo neutrals code (Eirene)[6] (following atomic and molecular hydrogen and atomic carbon and helium). Since it is this code package that is being used for the design of ITER (Kukushkin, this conference), it is important that the code is benchmarked against current machines and that the physical processes underlying the compression and enrichment are understood.

Therefore the dependence of helium compression and enrichment on plasma density, heating power and transport coefficients are examined. These trends are then compared to ASDEX Upgrade results. Both in the code and in the experiment helium compression is observed to rise with increasing divertor density. Approaching detach- ment, a fall was obtained numerically which is associated with complete detachment under which conditions the experiment might have disrupted.

In the modelling, the issue arises of how to treat ELM effects and two approaches are taken: averaging over ELMs with somewhat larger transport coefficients than normally associated with $\mathrm{H}$ mode plasmas and doing time-dependent simulations including a simple ELM model. These two approaches are seen to give broadly similar results, probably because the timescales for helium exhaust are much longer than the ELM timescale.

The geometry of the divertor can also play an important role and numerical results are also presented for the proposed new divertor on ASDEX Upgrade, as well as some preliminary modelling for JET.

\section{Methodology}

The simulations were done on a 96x24 (poloidal $\mathrm{x}$ radial) grid following all of the charge states of $\mathrm{H}$ (or D), $\mathrm{C}$ and $\mathrm{He}$ in $\mathrm{B} 2$, and $\mathrm{H}, \mathrm{H}_{2}, \mathrm{C}$ and He neutrals in Eirene. The "core" hydrogen density (about $1 \mathrm{~cm}$ in from the separatrix) was varied to give the density scan. The input power (equally divided between electrons and ions) was also set on this same boundary and varied for the power scan. Standard sheath boundary conditions were set at the targets and decay length boundary conditions on the edges of the scrape-off layer and the private flux region. A trace amount of He was specified by setting a constant density on the "core" boundary. The $\mathrm{C}$ was generated self-consistently by physical and chemical sputtering (the latter at a constant yield of 1\%). The base transport coefficients were that the parti- 
cle diffusivity (for all charge states) was $1 \mathrm{~m}^{2} \mathrm{~s}^{-1}$ and the electron and ion thermal diffusivities were $2 \mathrm{~m}^{2} \mathrm{~s}^{-1}$. In the transport variation simulations, these transport coefficients were multiplied by a constant.

The ELMs were simulated on a deeper, 96x36, grid and the base transport levels used were a tenth those in the steady state calculations. For a short time the transport coefficients were increased substantially (to a value higher than that used in the steady state calculation) in the outer part of the main plasma and the scrape-off layer, and the time-dependent behaviour followed.

This work builds upon earlier work done with B2-Eirene simulating compression[7] and ELMs [8] for the earlier, more open, divertor configuration of ASDEX Upgrade (see [9] for the experimental results).

\section{Results}

\subsection{Power and density scan}

Figure 1a shows the compression of helium versus the neutral flux density seen at the pump plenum as a function of plasma density and input power. Data from the experiment under Ohmic conditions is also shown[10]. The basic pattern at each input power is the same: the compression rises with increasing plasma density (which also, at least until full detachment, implies increasing flux density at the pump plenum). Close to detachment the compression starts to fall, and then, in the code at least, the flux density at the pump plenum also falls with increasing density. This gives rise to the characteristic shape.

Figure 1b shows the compression of hydrogen for the same variations of plasma conditions. Here similar behaviour as for the $\mathrm{He}$ is seen, though the increase of compression with neutral flux density is less steep.

The resulting enrichment is shown in figure 1c. This is a somewhat more complicated figure, being the ratio of the data shown in figure $1 \mathrm{a}$ to the data in figure $1 \mathrm{~b}$. The values should be compared to the 0.2 that would be required for ITER. The code underestimates the enrichment because it underestimated the numerator (the helium enrichment) and overestimated the denominator (the hydrogen compression) in the expression for the enrichment.

The reason for the underestimate of the helium compression at low densities is not fully understood: possible factors include kinetic effects not well treated by B2[11] and a possible breakdown
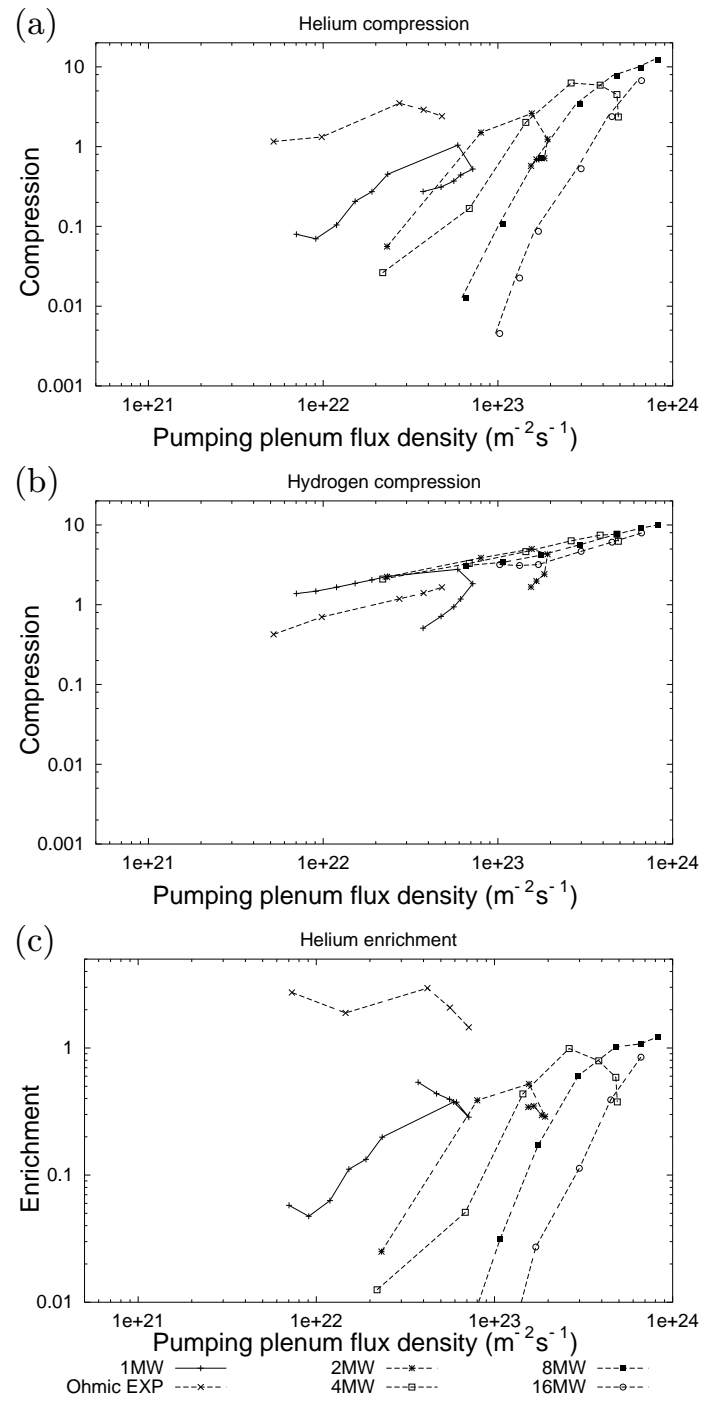

Figure 1. Helium (a) and hydrogen (b) compression, and helium enrichment (c) versus pumping plenum flux density (in particles $m^{-2} s^{-1}$ ).

of the technique used on the experiment to estimate the compression.

\subsection{Transport scan}

In the experiment the transport coefficients change with density and heating power in a manner that is not yet fully understood (but see Kim et al., this conference). To explore the effects of a variation in the transport coefficients, a scan was made for two densities for $1 \mathrm{MW}$ of input power. Despite changing the transport coefficients over a range of 0.125 to 4 times the basic value, the net 


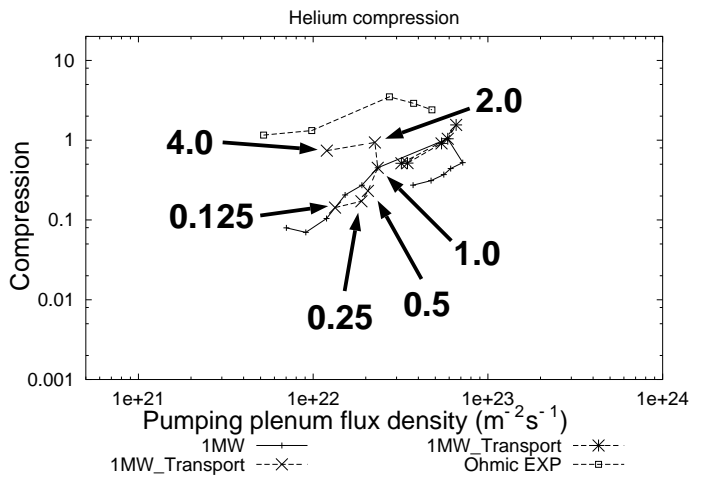

Figure 2. Helium compression versus pumping plenum flux density (in particles $m^{-2} s^{-1}$ ) showing the effects of varying the transport coefficients.

effects were small, as shown in figure 2 .

\subsection{Pumping scan}

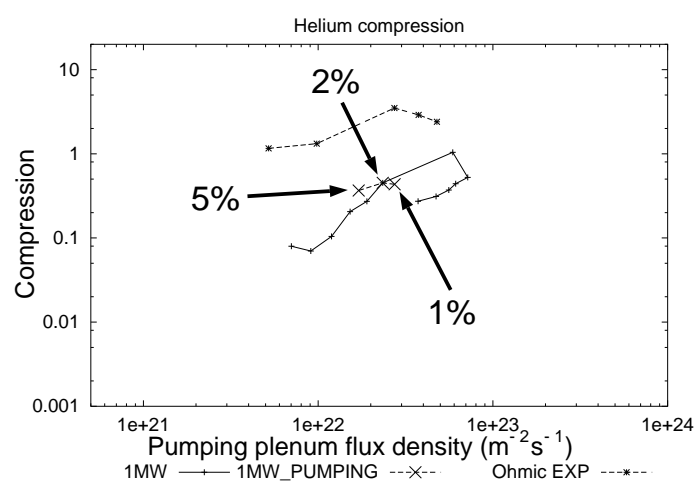

Figure 3. Helium compression versus pumping plenum flux density (in particles $m^{-2} s^{-1}$ ) showing the effects of varying the amount of pumping.

The amount of pumping would also be be expected to change the compression, though figure 3 shows a small effect despite a change of the pumping at the pump surface over a range of 5 (1\%, base $2 \%$ and $5 \%$ pumping efficiency at the entrance to the pump duct).
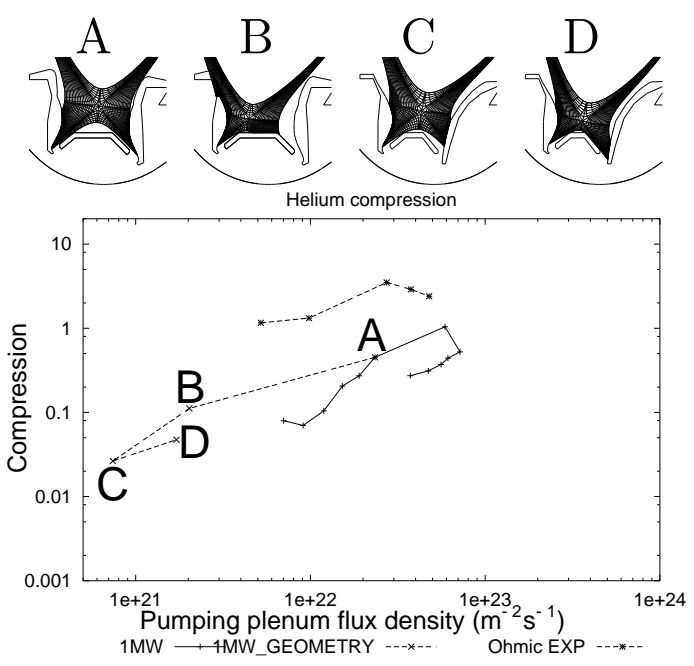

Figure 4. Helium compression versus pumping plenum flux density (in particles $m^{-2} s^{-1}$ ) showing the effects of varying the geometry.

\subsection{Geometry variation}

The geometry of the divertor can have a large effect. In figure 4 the helium enrichment for the standard ASDEX Upgrade case, A, is shown, as well as a high triangularity equilibrium which has the outer strike point on the roof baffle, B. Since the ASDEX Upgrade experimental programme is placing an increasing emphasis on higher triangularity, the divertor will be changed as shown for $\mathrm{C}$ and D. For the same conditions of input power and density, the standard case shows higher compression and significantly higher pump plenum flux density.

\subsection{ELMs}

In the previous simulations the compressions were calculated by steady state simulations using L-mode or ELM-average H-mode transport coefficients. Figure 5 shows the time dependent compression of helium. The basic result is the same as for the steady state calculations.

\subsection{JET}

Preliminary results of modelling of the helium compression for JET are shown in figure 6, under the assumption that the transport coefficients on JET and ASDEX Upgrade are the same. While we have no supporting evidence for this, it does allow a somewhat simpler comparison of the simulation results for the two machines showing the 


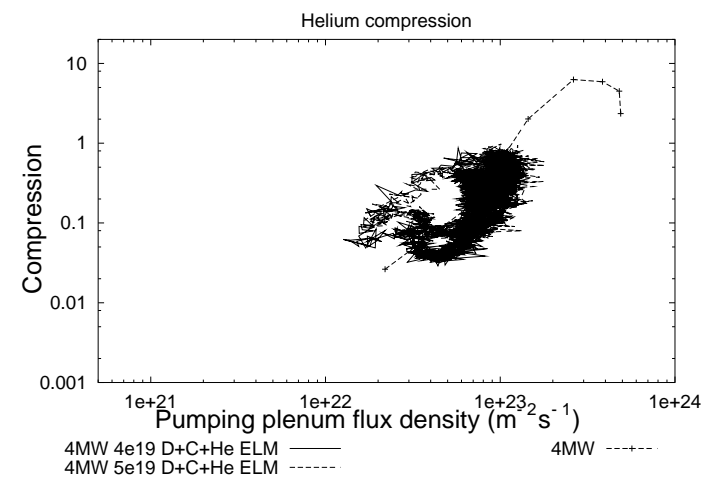

Figure 5. Helium compression versus pumping plenum flux density (in particles $m^{-2} s^{-1}$ ) showing the effects of ELMs compared to the $4 \mathrm{MW}$ case of figure 1a.

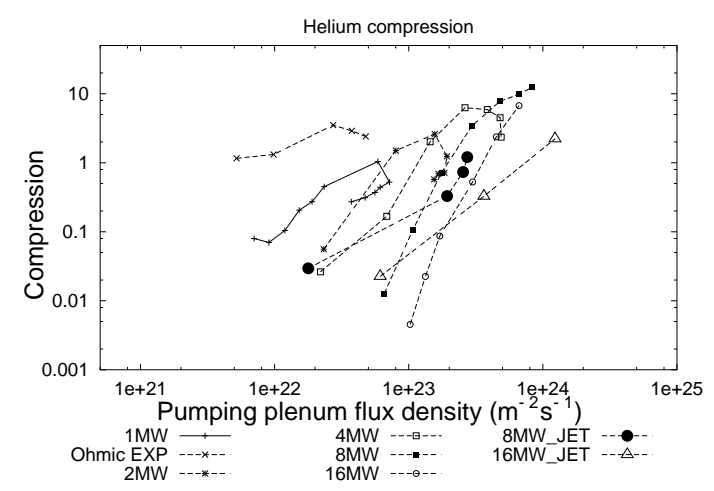

Figure 6. Preliminary helium compression for JET using the same transport coefficients that were used for the ASDEX Upgrade simulation.

effects of size and shape. The compressions for the two machines are in the same ballpark under these assumptions.

\section{Discussion}

The code reproduces the experimental observed trends of helium and hydrogen compression, though quantitative differences remain. For a broad range of densities and input powers sufficient compression and enrichment of helium occurs. There is, however, a minimum edge density, below which the helium would not be adequately pumped. This could pose problems for future advanced tokamak scenarios unless they can be extended to higher edge densities.

\section{REFERENCES}

1. KRASHENINNIKOV, S. et al., Nuclear Fusion 31 (1991) 1455.

2. SCHNEIDER, R. et al., J. Nucl. Mater. 196198 (1992) 810.

3. REITER, D., J. Nucl. Mater. 196-198 (1992) 80.

4. BRAAMS, B. J., Computational Studies in Tokamak Equilibrium and Transport, $\mathrm{PhD}$ thesis, Rijksuniversiteit, Utrecht, Nederland., 1986.

5. BRAAMS, B. J., A multi-fluid code for simulation of the edge plasma in tokamaks, Technical Report 68, Next European Torus, 1987.

6. REITER, D. et al., J. Nucl. Mater. 220-222 (1995) 987, PSI 94 Mito.

7. COSTER, D. P. et al., J. Nucl. Mater. 241243 (1997) 690.

8. COSTER, D. et al., B2-eirene modelling of ELMs on ASDEX Upgrade, in Proc. of the 21th European Conference on Controlled Fusion and Plasma Physics, Montpellier, 1994, edited by JOFFRIN, E. et al., volume 18B, part II, pages 846-849, Geneva, 1994, EPS.

9. BOSCH, H.-S. et al., Plasma Phys. Controlled Fusion 39 (1997) 1771.

10. ULLRICH, W., Helium transport in asdex upgrade, Laborbericht 1/321, IPP Garching - Doktorarbeit Universität Augsburg, Juli 1999.

11. REISER et al., this conference. 